\title{
DEVELOPMENT AND EVALUATION OF 'NECK PAIN AND FUNCTIONAL LIMITATION SCALE': A VALIDATION STUDY IN THE ASIAN CONTEXT
}

JOSEPH HENRY LEONARD, CHAN POH CHOO, MOHD. RIZAL ABDUL MANAF', ZALEHA MD ISA ${ }^{1}$, NOR AZLIN MOHD NORDIN, SRIJIT DAS²

\section{ABSTRACT}

BACKGROUND: There is a paucity of literature on validated outcome measurement tools for evaluation of neck pain and related disability in the Asian context. AIM: The main aim of the present study was to design a new tool called neck pain functional limitation scale (NPFLS) for measuring disability related to neck pain and observe its reliability, concurrent validity and criterion validity. SETTING AND DESIGN: This study was performed at the institutional hospital. MATERIALS AND METHODS: A total of 157 subjects (neck pain group) and 25 control subjects (control group) without neck pain were recruited for this study. NPFLS was framed as a new tool for this study, which consisted of 5 domains - pain intensity, activities of daily living, social activities, functional activities and psychological factors. Neck Bournemouth questionnaire (NBQ) was used as a gold standard to measure the concurrent validity and criterion validity of the N PFLS. STATISTICAL ANALYSIS: Criterion validity and concurrent validity between the neck Bournemouth questionnaire (NBQ) and NPFLS scores were tested statistically using Mann-Whitney $U$ test and Spearman correlation test. The reliability was tested by examining the internal consistency to calculate the Cronbach's alpha value for each item in NPFLS. RESULTS: No significant difference between NPFLS and NBQ was observed using Mann-Whitney $U$ Test, with $P$ value greater than $0.05(P=0.557)$. Besides that, NPFLS had a high concurrent validity $(r=0.916)$ and good internal consistency with high Cronbach's alpha value of $(r=0.948)$, which demonstrated strong correlation between the items of NPFLS and NBQ. CONCLUSION: N PFLS demonstrated good reliability, high concurrent validity and criterion validity in this study. NPFLS can be used to assess neck pain and disability among patients with neck pain.

Key words: Design, neck, outcome measure, pain, questionnaire, reliability, validity DOI: $10.4103 / 0019-5359.57645$

PMID: 19901483

Physiotherapy Program, Faculty of Allied Health

Sciences, Departments of ${ }^{1}$ Community Health and

${ }^{2}$ Anatomy, Universiti Kebangsaan Malaysia Medical

Centre (PPUKM), Kuala Lumpur, Malaysia

\section{Correspondence:}

Dr. Joseph H. Leonard

Universiti Kebangsaan Malaysia,

Jalan Raja Muda Abdul Aziz, Kuala Lumpur, Malaysia 50300

E-mail: leonardjoseph85@ hotmail.com

\section{INTRODUCTION}

Neck pain is one of the most common problems in the population, which affects approximately $67 \%$ of individuals at some point of time in their lifetime. ${ }^{[1]}$ Regarding the 12-month prevalence of neck pain, previous research studies have 
reported it to range between $30 \%$ and $50 \% .^{[1-2]}$ Although it is not life threatening, it can cause a sense of being unwell and substantial level of disability due to pain and neck stiffness. ${ }^{[2]}$ This disability can affect the physical functioning of the patients, leading to sickness behavior and activity restrictions. ${ }^{[3-4]}$ In the general population, the 12-month prevalence of activity-limiting pain has been reported to vary from $1.7 \%$ to $11.5 \% \cdot{ }^{[1]}$

The severity of neck pain and the related disability can affect daily social and functional activities, which may even involve emotional and psychological aspects. ${ }^{[5-11]}$ Thanks to the approval of the International Classification of Functioning, Disability and Health (ICF) by the World Health Assembly in 2001, neck pain and related consequences could be clearly understood and evaluated by a universal conceptual model termed as 'biopsychosocial health,' which integrated the biomedical and societal models of functioning and disability. ${ }^{[12]}$ Hence, the outcome measures for any disease that predict the disease progress and response should carefully consider the biopsychosocial model involved in the evaluation of the disease process. $^{[12]}$

A few disease-specific outcome measurement tools that are available for assessing neck pain include neck disability index, neck pain and disability scale, Copenhagen neck functional disability scale, Northwick Park pain questionnaire, patient-specific functional scale self-reports with neck dysfunctions and the North American Spine Society cervical spine outcome assessment instrument. ${ }^{[8-10,13-15]}$ Interestingly, all the abovementioned tools were developed considering the psychosocial aspects of western culture and were validated in the western context. These tools may have cultural bias and may be unsuitable for use in the Asian context because of the differences in the local cultural practices. ${ }^{[16]}$ Hence there was a need to develop a disease-specific outcome measurement tool for neck pain that reflects the local cultural practice. ${ }^{[16]}$ Thus NPFLS was designed as a new outcome measurement tool to evaluate neck pain.

It is important that the newly developed outcome measurement tool must demonstrate reliability (consistency), validity (trueness) and responsiveness (the ability to detect change). ${ }^{[17]}$ If the results of the tool are valid, then it should measure the trait for which it was designed (content), be correlated to other measures of that trait (criterion) and must differentiate between the group with disease and the one without it. Hence the main aim of the present study was to create a new outcome measurement tool, NPFLS, in order to assess the disability involved in neck pain and to report its reliability, concurrent validity and criterion validity.

\section{MATERIALS AND METHODS}

The present cross-sectional study examined the validity of the NPFLS. This study was performed in the Physiotherapy Department of an institutional hospital. A total of 157 subjects diagnosed with neck pain by orthopedic specialists and 25 subjects without neck pain were recruited for this study. The inclusion criteria for recruitment of the 157 subjects included neck pain for more than a month; and history of cervical disk herniation, cervical 
spondylosis, cervical spine fracture and postsurgical conditions of neck. Patients with neck pain and lower back pain, patients with migraine with or without aura along with neck pain and patients with neck pain with history of headaches were excluded from the study.

The control subjects were recruited from the patients who attended the physiotherapy clinic with other musculoskeletal problems that were not related to the neck and shoulder. The purpose and procedure of the study were explained to all the subjects by a physiotherapist, and informed written consent was obtained before testing of the tools. NPFLS and NBQ were administered to all the subjects by the same physiotherapist. In order to minimize the learning effects and bias in the administration of the tools, the tools were administered randomly by asking the subject to pick a card, behind which the name of the particular tool was written (either NPFLS or NBQ). All the tests were carried out by the same physiotherapist. Prior ethical approval was obtained from the institution.

\section{Framing of NPFLS}

The NPFLS was created to assess the disability related to neck pain. This tool consisted of 5 main domains, viz., pain intensity, activities of daily living, social activities, functional activities and psychological factors. Each domain had 4 sub-items that reflected the psychometric properties of that particular domain, with a total of 20 questions in NPFLS. The subject had to circle only 1 answer in each question. Scores for each question ranged from 0 to 5 . The highest score indicated greater disability.

The framing of NPFLS involved several steps.
First, a review of the literature on neck pain, disability scales and questionnaire-designing was carried out in order to find out a list of items to be considered in NPFLS. Secondly, a focused group analysis using a patient-specific technique was carried out to collect details regarding patient's perception, beliefs and attitude about neck pain and its impact on their daily life; these were collected as sub-items by structured interview among a different group of patients with neck pain. Next, Team A, which consisted of 4 senior physiotherapists, was asked to give its expert opinion on disability caused by neck pain and compare the items from the literature review with patient's selfreported items. An expert opinion from a statistician was also obtained for the scoring scale.

Thus, an initial NPFLS questionnaire (consisting of 36 items) comprising of summarized items was prepared and was sent to Team B for a review. This team consisted of 4 other senior physiotherapists specialized in musculoskeletal practice and a senior medical practitioner. Face validity was obtained from Team B, which came out with the final version of NPFLS (20 items) after removing the unpopular items and adjusting the tool for domain and syntax. The main domains and each sub-item under every main domain in NPFLS were presented as follows:

Domain 1 - Pain intensity: It consisted of 4 questions, which included neck pain rating, duration of neck pain, ability to manage neck pain and ability to tolerate neck pain.

Domain 2 - Activities of daily living (ADL): It consisted of 4 questions, which included dressing, head turning, television-viewing and carrying things. 
Domain 3 - Social activities: It consisted of 4 questions, which were related to shopping, family relationships and interactions, traveling and recreational activities.

Domain 4 - Functional activities: It consisted of 4 questions, which included reading, using phone, looking up to search things above head level and work.

Domain 5 - Psychological factors: It consisted of 4 questions, which were related to sleeping, ability to concentrate, feeling of anxiousness and feeling of depression.

The above-mentioned 5 main domains and the sub-items under each domain reflected the biopsychosocial status of health, which can be influenced by the presence of any disease that can cause disability in the normal functioning patient. The term biopsychosocial is a collective manifestation of functional, social and psychological events that can be influenced by the presence of neck pain. ${ }^{[12]}$

\section{Neck Bournemouth questionnaire}

NBQ consisted of 7 core items, which included pain intensity, disability in activities of daily living (ADL), disability in social activities, the emotional dimensions of anxiety and depression, the cognitive aspects of fearavoidance behavior and pain control. Each item score ranged from 0 to 10 , and the total scores were calculated. The higher score indicated greater disability. Since NBQ consisted of 5 main domains, namely, pain intensity, activities of daily living ( $A D L)$, social activities, functional, emotional and psychological factors, it could be used to compare the criterion validity and concurrent validity of NPFLS. NBQ was tested and found to be valid and reliable in patients with nonspecific neck pain. ${ }^{[7]}$ Since NBQ was valid and reliable, it was used in this study to test the psychometric properties of NPFLS.

\section{Statistical methods}

The collected data was analyzed with SPSS version 16.0. Descriptive statistics was used to analyze the demographic characteristics of the subjects. The continuous data was subjected to normality tests and revealed that it was not normally distributed. Hence nonparametric statistical tests were used to analyze the data. The criterion validity between the NPFLS and NBQ was assessed by Mann-Whitney test. The concurrent validity between the two tools was analyzed through Spearman correlation test. The agreement between the NPFLS and NBQ was assessed by weighted kappa statistics with $95 \%$ confidence interval. If the kappa coefficient value ranged between 0.6 and 1.0 , it was considered as good agreement between the NBQ and NPFLS. The reliability of NPFLS was tested by examining the internal consistency to calculate the Cronbach's alpha value of each item.

\section{RESULTS}

Out of 157 patients with neck pain, 66 were males and 91 were females. The average age of these subjects was 47.18 years (minimum, 15 years; and maximum, 78 years). Table 1 shows the demographic characteristics of the subjects with neck pain who participated in this study.

The results of criterion validity analysis between NPFLS and NBQ showed that there was no difference between the two tools $(P>0.05$, $P=0.557)$. Spearman's correlation results for concurrent validity between the scores of NBQ 
Table 1: Summary of demographic characteristics of the subjects

\begin{tabular}{lc}
\hline & $N(\%)$ \\
\hline Gender & \\
Male & $66(42.0)$ \\
Female & $91(58.0)$ \\
Age Group & \\
$11-20$ & $2(1.3)$ \\
$21-30$ & $34(21.7)$ \\
$31-40$ & $15(9.6)$ \\
$41-50$ & $30(19.1)$ \\
$51-60$ & $40(25.5)$ \\
$61-70$ & $26(16.6)$ \\
$71-80$ & $10(6.4)$ \\
Pain Duration Group & \\
$60-120$ days & $147(93.7)$ \\
$121-180$ days & $2(1.3)$ \\
$181-240$ days & $5(3.2)$ \\
$241-300$ days & $0(0.0)$ \\
$301-360$ days & $3(1.9)$ \\
Diagnosis & \\
Cervical Spine Fracture & $1(6.0)$ \\
Cervical Spine Stenosis & $2(1.3)$ \\
Cervical Spondylosis & $50(31.8)$ \\
Neck Pain with Unknown Cause & $93(59.2)$ \\
Prolapsed Intervertebral Disc & $7(4.5)$ \\
Whiplash & $4(2.5)$ \\
Occupation & $11(7.0)$ \\
Armed Forces & $18(11.5)$ \\
Housewife & $4(2.5)$ \\
Nursing & $28(17.8)$ \\
Office Worker & $23(14.6)$ \\
Professional & $9(5.7)$ \\
Student & $8(5.1)$ \\
Teaching & $44(28.0)$ \\
Unemployed & $7(4.5)$ \\
Worker & $5(3.2)$ \\
Others & \\
\hline & \\
\hline &
\end{tabular}

and NPFLS showed that there was a strong correlation between NBQ and NPFLS scores, with correlation coefficient $r=0.916$.

The internal consistency, as measured by the Cronbach's alpha coefficient, was more than 0.7 in all the items in NPFLS (overall value, 0.948). Intra-class correlation coefficient analysis between NPFLS and NBQ showed that the average measure of intra-class correlation coefficient value was very strong and highly significant $(r=0.962, P<0.0005)$.

\section{DISCUSSION}

NPFLS is a newly designed outcome measurement tool meant for evaluation of neck pain and related disability. The main aim of this study was to create NPFLS as an outcome measurement tool for neck pain and to examine the concurrent validity and construct validity, along with the reliability of NPFLS. However, before discussing the validity and reliability aspects of the NPFLS, it is important to discuss the construction and contents of NPFLS. The basic design of NPFLS considered the 'biopsychosocial health' aspects as a conceptual model, recommended by the International Classification of Functioning Disability and Health (ICF). ${ }^{[12]}$

Recently, research on neck pain had shifted its focus away from the signs and symptoms. Rather, importance was laid on the specific effects of the symptoms on the patient's functioning and daily life. ${ }^{[14]}$ The above facts were supported by a previous study which looked at neck function, physical function more holistically and at psychological function, which supported the reasons for inclusion of the multidimensional domains in the construction of NPFLS. ${ }^{[19]}$ Therefore, the main 5 domains of NPFLS are pain intensity, activities of daily living, functional domain, social domain and psychological domain. Each domain has four sub-items, which makes for a total of 20 items in NPFLS. The 5 main domains of the NPFLS and the sub-items were framed from items generated from neck pain-focused group interviews, items generated from literature review and items generated from the information received from clinical specialists dealing with neck pain.

Previous studies that dealt with neck pain had identified rating of pain, duration of neck pain, 
ability to manage neck pain and the ability to tolerate neck pain as the important factors which were to be considered while assessing pain among patients with neck pain. ${ }^{[7-9]}$ Hence the pain intensity domain was supported with sub-items which included neck pain rating, duration of neck pain, ability to manage neck pain and ability to tolerate neck pain.

The physical factors associated with neck pain included heavy lifting, monotonous work tasks, static work posture, vibrations, repetitive jobs and a high work pace. ${ }^{[20]}$ However, the patients from the 'focused interview' group identified activities such as turning head during driving, viewing television, carrying things and dressing as the main problems that resulted from their neck pain. These were included as items under the domain of activities of daily living.

Previous studies had pointed out that neck pain can affect social factors, which include shopping, family relationships and interactions, traveling and recreational activities..$^{[7-8]}$ Hence these items were included to assess the effects of neck pain and disability within the social context. In the patient-specific focused group interview for neck pain, majority of the patients complained of neck pain while reading a newspaper or a book in a flexed neck position. Furthermore, previous studies had listed using phone, looking up to search things above head level and daily work as the main functional activities affected due to neck pain..$^{[9]}$ Hence the functional activities domain consisted of 4 questions, which included reading, using phone, looking up to search for things above head level and normal daily work activities.

Previous research had shown a direct correlation between the pain level and the attention paid to psychosocial distress, especially anxiety and depression. ${ }^{[11]}$ Other psychological factors affected by neck pain were disturbed sleep due to pain, lack of ability to concentrate and focus, feelings of anxiety and depression. ${ }^{[7-10]}$ Disturbed sleep and sense of depression were commonly cited by the individuals in the neck pain focus group as disabling factors related with neck pain. Therefore, the psychological domain consisted of 4 questions, which included those related to sleeping, ability to concentrate and focus, and feelings of anxiousness and depression.

The methodology to design the NPFLS is also discussed. The designing process of the NPFLS considered the patient-specific techniques (neck pain focus group) to come out with their own set of problems or items which had been matched with the fixed items obtained by a scientific literature search process. The advantage of this method was that it identified the problems or issues relevant to each individual and was consistent with the approach to patient evaluation commonly used in clinical practice. ${ }^{[21]}$ This was important because the traditional fixed-items methods employed to design a questionnaire may constrain the scope of evaluation and include questions irrelevant to some patients or may not include issues of importance to some patients. ${ }^{[19]}$ Therefore, the method adopted to create NPFLS overcame the design flaws in an attempt to obtain more accuracy.

It is for the same reasons stated above that a patient-specific scale was not considered for simultaneous validation along with NPFLS and NBQ. Moreover, the level of statistical 
correlation between patient-specific scales and fixed-items questionnaires was always found to be only moderate due to the limitation in the standardization of the contents in the context of patients' perceptions of their problems. ${ }^{[22-23]} A$ previous study had mentioned that the patientspecific scale may be perceived differently by different persons, especially those concerning to the domains of social, psychological and emotional functions. ${ }^{[21]}$ Therefore, this study considered patient-specific techniques to elicit information in framing of NPFLS, along with the items supported by evidence-based practice in the field of neck pain, but it did not include a simultaneous patient-specific scale in the validation process. Hence the validation of the NPFLS was decided to be carried out with a matched gold standard which reflected the same properties of NPFLS.

While developing a questionnaire, it is important to include in the research design to add an established measure with proven validity. ${ }^{[24]}$ Therefore, NBQ tool with established validity and reliability was added in this study to test the psychometric properties of NPFLS. ${ }^{[7]}$ The results of this study revealed that NPFLS was an internally consistent and valid measure that could be used to distinguish patients with neck pain from other healthy volunteers.

Content validity of NPFLS was attempted by adjusting the 5 domains and syntax of each question with consideration of patients' comments and experts' opinions supported by scientific information from literature. Regarding content validity, any instrument designed to measure such a construct must tap each of its constituent dimensions. ${ }^{[25]}$ NPFLS consisted of 5 main domains in which its psychometric properties matched with those of NBQ. Therefore, in this study, the psychometric properties of NPFLS were matched with the established psychometric properties of NBQ in order to examine the criterion validity and concurrent validity of NPFLS.

Criterion validity was established by measuring the sensitivity and specificity of NPFLS in this study. The sensitivity of a test was the extent to which it identified those patients who in fact had the disease, whereas the specificity of a test was the extent to which it failed to identify those without the disease. ${ }^{[26]}$ The results of the study showed that NPFLS had good criterion validity $(P=0.557)$.

Concurrent validity was established by comparing the performance of NPFLS with that of NBQ in this study. The test-retest reliability of NBQ was 0.65 with acceptable construct validity. ${ }^{[7]}$ The NPFLS produced data that was in accordance with $\mathrm{NBQ}$, and therefore, it was deemed to be a valid measure. In this study, there was a good correlation between NPFLS and NPFLS total scores by using Spearman's correlation. The correlation coefficient value was 0.916 , which meant that the scores were highly correlated to each other.

Cronbach's alpha was used to measure reliability and inter-correlation of the items of NPFLS in this study. Cronbach's alpha could take a value between 0 and 1 , where 0 indicated that none of the items was related to another and 1 indicated that all items were the same. ${ }^{[27]}$ If the reliability coefficient was 0.80 or higher, it was considered high or good reliability. ${ }^{[17]}$ In this study, NPFLS had high Cronbach's alpha value $(r=0.948)$ and showed that it had good 
reliability. Furthermore, the intra-class correlation coefficient between NPFLS and NBQ was high $(r=0.962)$, which showed that the psychometric properties of NPFLS were highly reliable and comparable to those of NBQ.

In terms of comparing the performance of NPFLS with regard to the psychometric properties of the other available tools to assess neck pain, NPFLS performed better in reliability scores. For example, the reported Cronbach's alpha value was 0.88 for Copenhagen neck functional disability scale, whereas the obtained Cronbach's alpha value for NPFLS was 0.948. ${ }^{[10]}$ Similarly, the reported Cronbach's alpha value for neck disability index ranged from 0.6 to 0.7 , which was lesser compared to that for NPFLS. Hence it could be claimed that NPFLS performed better when compared to some of the other available tools to assess neck pain.

As Malay is the official language in Malaysia and the general hospitals in Malaysia do not have a standardized and validated tool for measuring neck pain and disability, there was a need to have an outcome measurement tool in Malay language to evaluate neck pain. Therefore, it is the opinion of the researchers of this study that efforts should be made to report on the validation of the English version of the newly designed NPFLS, which would be translated into Malay language later and validated in a future study for local usage in Malaysia.

This study has some strength in that the subjects with neck pain recruited in the study represented a variety of cervical problems, ranging from cervical spondylosis to cervical disc prolapse. The recruitment of patients with both nonspecific neck pain and specific neck pain (a structural damage to the neck tissue) allowed the applicability of NPFLS to various types of neck pathologies. Similarly, the age range of the patients who participated in this study also consisted of both younger and older age groups of patients. Hence it could be said that NPFLS may be applied to different age groups. Strength may be accounted for by the process of designing the NPFLS. Previous tools related with neck pain configured questionnaire items based on literature reviews and were compared with a patient-specific tool. ${ }^{[7-9]}$ The NPFLS was designed by collecting information using patientspecific methods, expert opinions, along with the items supported scientifically through literature search.

The researchers also would like to humbly admit some limitations of this study. Firstly, the subjects for this study were recruited from one particular hospital and only from the outpatient department. Secondly, NPFLS was in its initial stages of validation. Validity aspects such as predictive validity, responsiveness to change, test-retest reliability, factorial analysis, etc., are being considered for future studies before NPFLS could be translated into Malay language for the local population.

\section{CONCLUSION}

The present study supported the validity of NPFLS because the performance of NPFLS was comparable to the established standards of NBQ. The study results suggested that NPFLS was a highly reliable outcome measurement tool to evaluate neck pain and underlying disability.

\section{ACKNOWLEDGMENT}

The authors wish to thank and acknowledge the 
facilitation rendered by the "Publication Centre for Health Cluster, Universiti Kebangsaan Malaysia" for providing the facilities and guidance for completing this manuscript. The authors also wish to thank all the senior physiotherapists who contributed to this study by helping in framing the NPFLS. We would also like to thank the Physiotherapy Department for the assistance provided in collecting data for this study.

\section{REFERENCES}

1. Cote P, Cassidy DJ, Carroll L. The Saskatchewan health and back pain survey: The prevalence of neck pain and related disability in Saskatchewan adults. Spine 1998;23:1689-98.

2. Crombie IK, ed. The epidemiology of pain. Seattle; IASP Press: 1999.

3. Borghouts JA, Koes BW, Bouter LM. The clinical course of non-specific neck pain: A systematic review. Pain 1998;77:1-13.

4. Cagnie B, Danneels L, Tiggelen DV, DeLoose V, Cambier D. Individual and work related risk factors for neck pain among office workers: A cross sectional study. Eur Spine J 2007;16:67986.

5. Côté P, Cassidy DJ, Carroll L. The prevalence of neck pain and related disability In saskatchewan adults. Spine 1998;23:1689-98.

6. Borghouts JA, Koes BW, Vondeling H. Cost-ofillness of neck pain in the netherlands in 1996. Pain 1999;80:629-36.

7. Bolton JE, Humphreys BK. The bournemouth questionnaire: A short-form comprehensive outcome measure II: Psychometric properties in neck pain patients. J Manipulative Physiol Ther 2002;25:141-8.

8. Vernon H, Hagino C. 1987. Vernon H, Mior S. The neck disability index: A study of reliability and validity. J Manipulative Physiol Ther 1991;14: 409-15.

9. Leak AM, Cooper J, Dyer S, Williams KA,
Turnerstokes L, Frank AO. The northwick park neck pain questionnaire devised tto measure neck pain and disability. Br J Rheumatol 1994;33: 469-74.

10. Jordan A, Manniche C, Mosdal C, Hindsberger C. The copenhagen functional disability scale: A study of reliability and validity. J Manipulative Physiol Ther 1998;21:520-7.

11. Blozik E, Laptinskaya D, Herrmann-Lingen C, Schaefer $\mathrm{H}$, Kochen MM, Himmel W, et al. Depression and anxiety as major determinants of neck pain: A cross-sectional study in general practice. BMC Musculoskelet Disord 2009;10:13.

12. Stucki G, Boonen A, Tugwell P, Cieza A, Boers $M$. The world health organisation international classification of functioning, disability and health: A conceptual model and interface for the OMERACT process. J Rheumatol 2007;34:600-6.

13. Wheeler AH, Goolkasian P, Baird AC, Darden BV $2^{\text {nd }}$. Development of the neck pain and disability scale: Item analysis, face, and criterion-related validity. Spine (Phila Pa 1976) 1999;24:1290-4.

14. Pietrobon R, Coeytaux RR, Carey TS, Richardson WJ, DeVellis RF. Standard scales for measurement of functional outcome for cervical pain or dysfunction: A systematic review. Spine 2002;27:515-22.

15. BenDebba M, Heller J, Ducker TB, Eisinger JM. Cervical spine outcomes questionnaire: its development and psychometric properties. Spine (Phila Pa 1976) 2002;27:2116-23.

16. Ali YM, Gill R. A psychometric assessment of the malay version of Meyer and Allen's organizational commitment measure. Malaysian Manage Rev 1999;34:32-9. Available from: http://myais.fsktm. um.edu.my.

17. Joy CM, Mule M. Concurrent validity of the NK hand dexterity test. Physiother Res Int 2001;6: 83-93.

18. Kochhar PH, Rajadhyaksha SS, Suvarna VR. Translation and validation of brief patient health questionnaire against DSM IV as a tool to diagnose major depressive disorder in Indian 
patients. J Postgrad Med 2007;53:102-7.

19. En MC, Clair DA, Edmondston SJ. Validity of the neck disability index and neck pain and disability scale for measuring disability associated with chronic, non-traumatic neck pain. Man Ther 2009;14:433-8.

20. Croft PR, Lewis M, Papageorgiou AC, Thomas E, Jayson MI, Macfarlane GJ. Risk factors for neck pain: A longitudinal study in the general population. Pain 2001;93:317-25.

21. Jolles BM, Buchbinder R, Beaton DE. A study compared nine patient-specific indices for musculoskeletal disorders. J Clin Epidemiol 2005;58:791-801.

22. Hoving JL, O'Leary EF, Niere KR, Green S, Buchbinder R. Validity of the neck disability index, Northwick Park neck pain questionnaire, and problem elicitation technique for measuring disability associated with whiplash-associated disorders. Pain 2003;102:273-81.

23. Westaway MD, Stratford PW, Binkley JM. The patient-specific functional scale: Validation of its use in persons with neck dysfunction. J Orthop Sports Phys Ther 1998;27:331-8.

24. Rattray J, Jones MC. Essential elements of questionnaire design and development. J Clin Nurs 2007;16:234-43.

25. Borsboom D, Mellenbergh GJ, van Heerden J. The concept of validity. Psychol Rev. 2004;111:1061-71.

26. Haynes SN, Lench HC. Incremental validity of new clinical assessment measures. Psychol Assess. 2003;15:456-66.

27. Bland JM, Altman DG. Cronbach's alpha. BMJ 1997;314:572.

Source of Support: Nil. Conflict of Interest: None declared. 